\section{September Editorial Clarified}

\section{To the Editor:}

My first reaction to Dr. Charles S. Bryan's editorial in the September issue of Infection Control ${ }^{1}$ was that it offered "an interesting approach." But the more I thought about it the more something bothered me. In fact, a lot of things bothered me. I have decided that his concept is just another example of the "Doctrine of Divinity" that many doctors still seem to want to perpetuate.

First, Dr. Bryan is overlooking some basic elementary facts (yes, epidemiologic facts) about infection control in hospitals:

1) All hospitals have epidemiologically important bacteria as part of the ambient flora.

2) All patients should be considered susceptible to infections due to their immobility, possibly decreased nutritional status, invasive procedures, indwelling lines, antibiotic therapy, and the stress of hospitalization.

3) Colonization with this ambient flora occurs among physicians as well as among other patient care personnel and patients.

4) Physicians, also, unconsciously touch their noses, faces, and mouths, thereby possibly contaminating their own hands with colonizing flora. Physicians, also, can be shedders.

5) Washing between each patient contact is the highest standard possible-if you accept a lower standard to start with, your average will never be acceptable.

Second, I am unclear what the revisionist interpretation of Dr. Semmelweis has to do with Dr. Bryan's reason for lowering the standards for handwashing by physicians. Dr. Bryan's handwashing recommendations seem to reflect the underlying attitude of only needing to wash when hands are obviously soiled. Why should handwashing standards be any different for doctors than they are for other patient care personnel?

Finally, it is really a sad commentary if physician epidemiologists can only relate to physicians, and nurse epidemiologists can only relate to nurses. That's a lose-lose situation and surely the devil does indeed win.

If historical perspective is of interest to Dr. Bryan, perhaps he might find this look at the past, present, and future to be helpful. ${ }^{2}$

\section{REFERENCES}

1. Bryan CS: Of soap and Semmelweis (editorial). Infect Control 1986; 7(9):445-447.

2. Brown AF, Otterman JL: "The coccus-conscious" or "the conscious careful". Infect Control 1987; 8(1):34-35.

Joan L. Otterman, BSN, RN, CIC Nurse Epidemiologist Glendale Adventist Medical Center Glendale, CA

Dr. Charles Bryan responds to Joan Otterman's letter:

I thank you for expressing your thoughts and concerns. An adequate response requires a defense of the current CDC guidelines, an account of my own activities, and a clarification of a key aspect of the Semmelweis dilemma which may have been inapparent to Ms. Otterman, and perhaps to other nonphysician readers.

First, I can reassure Ms. Otterman that I, too, attach importance to all but the last of the "basic elementary facts." However, these seemed too well-known to require recitation in the editorial pages of Infection Control. Ms. Otterman's fifth "basic elementary fact" suggests either unfamiliarity or disagreement with the new CDC guidelines. ${ }^{2}$ These guidelines recognize that "the absolute indications for and the ideal frequency of handwashing are generally not known." They further stipulate that many routine patient care activities involving direct patient contact (such as taking a blood pressure reading) and most routine activities involving indirect patient contact do not require handwashing.

Second, I did not state in the editorial that handwashing guidelines for physicians should differ from those for other personnel, nor did I state that washing should be done only when hands are obviously soiled. I fail to see how Ms. Otterman gleaned either of these conclusions from my editorial. Apart from emergencies, "obvious soiling" of hands should be extremely rare since gloves should be worn when such circumstances are anticipated.

Finally, I did not state that physician epidemiologists should relate only to physicians and nurses only to nurses. I feel strongly that physicians can and should teach nurses and also that nurses can and should teach physicians. Both physicians and nurses should emphasize rational handwashing based on proper appreciation of epidemiologic principles. I came to this conclusion many years ago, based on making infection control rounds with our nursing staff.

For years, we have made the "fifteen second handwashing drill" a standard feature of these rounds. ${ }^{3}$ An account of this drill may be worthwhile. (Although some readers might consider the following to be somewhat chauvinistic, I defend the right to teach by the Socratic method). The dialogue usually goes as follows: Epidemiologist (to a newly arrived nursing student, chosen from the group assembled at the nursing station): What is the single most important infection control measure?

Student: Handwashing.

Epidemiologist: Good! And what is the recommended duration for routine handwashing at this hospital? Student (hesitating): I don't know. Epidemiologist: Come on, take a guess! Student: Three minutes. 
Epidemiologist: That's three minutes before and after each patient contact, right?

Student: Right.

Epidemiologist: So that the total duration of handwashing for each patient contact is six minutes.

Student (smiling): Right.

Epidemiologist: How many patients do you think the average nurse is assigned to for each shift?

Student: Well, maybe about eight.

Epidemiologist: And how many times do you think the average nurse will visit the average patient in the course of a nursing shift?

Student: Well, maybe about ten times. Epidemiologist: Let's see . . that's eight patients, ten visits per patient, and six minutes of handwashing per visit $\ldots 8 \times 10 \times 6=480 \ldots$ that's 480 minutes . . . divided by $60 \mathrm{~min}$ utes per hour . . . that's eight hours of handwashing per shift ... (feigning great surprise). You mean to tell me that the average nurse comes to work and does nothing but wash his or her hands for eight solid hours!? When is there time to take care of patients!? (All chuckle).

Epidemiologist: Well, I'm not trying to single you out. It seems that most new students don't know the recommended duration of handwashing at this hospital. (Turning to head nurse): What is the recommended duration of handwashing at this hospital?

Head nurse: Fifteen seconds.

Epidemiologist: That's right, 15 seconds. We find it important to try to develop a sense of the actual duration of those 15 seconds, so when I say 'go' I want you to rub your hands together for 15 seconds. Go! (All rub their hands together for 15 seconds while epidemiologist makes a few remarks about rubbing technique).

Epidemiologist: Stop. Now ... (making eye contact with each nurse, student, and physician present) ... I want you to tell me whether any of you really wash your hands that long before and after each patient contact. (Everyone nods no).

Epidemiologist: Well, this may sound like heresy but actually I don't think it's important to wash your hands before and after every patient contact. For example, if the patient is just in for diagnostic tests and you're only stick- ing your head in the door to find out why the buzzer went off, there's really no reason to wash your hands. But I think it's terribly important to wash your hands in situations in which you are likely to pick up bacteria or other 'germs' from taking care of a patient, or in which the patient is especially vulnerable to developing infection. Let's go over some of those situations... (There follows a review based to a large extent on the findings of the previous day's surveillance on the nursing unit).

Such interactions with our nursing staff convinced me of the soundness of the principles behind the new CDC guidelines long before these guidelines were published. Mindless "before and after every patient contact" handwashing should be replaced by rational handwashing based on understanding of the classic epidemiologic triad (reservoir-vector-susceptible host). As a corollary, handwashing frequency can and should vary among nursing units. In the intensive care unit (the thrust of my editorial), "washing before and after" applies to most patient contacts. On the psychiatry service, ordinary cleanliness suffices. Elsewhere, risk is intermediate. Frequency of handwashing should be based, at least in part, on appreciation of which patients are likely to be significant reservoirs of nosocomial pathogens and of which patients are especially susceptible hosts. Parenthetically, I agree with Ms. Otterman that it is sometimes best to err to the side of overemphasis and I therefore cling to "15 seconds" rather than the "10 seconds" of the new CDC guidelines. I also teach that one should always regard the hands as potential vectors and that both physicians and nurses should supplement handwashing with disposable rubber gloves in all high-risk situations.

By "Doctrine of Divinity," I assume that Ms. Otterman refers to the condescending attitude toward nurses attributed to many physicians. I am not altogether insensitive to this prob$\operatorname{lem}^{4}$ and suggest that Ms. Otterman give South Carolina nurses jurisdiction over my own case. However, Ms. Otterman appears to have missed the major point of my editorial: the need for physician epidemiologists to relate creatively not only to the nursing staff but also to physicians. Even on Mount Olympus, the gods had their own set of interpersonal problems. The Semmelweis dilemma-how to teach infection control measures to other physicians without offending them-still applies.

Hospital nurses answer primarily to administrators, and their day-to-day livelihood does not depend upon whether their colleagues especially like them. Physician epidemiologists-and I speak primarily of infectious diseases clinicians-live more precariously. Their consultative practice depends entirely upon the good will of their peers. They are not paid particularly well for their cognitive skills, and some even question their economic viability. 5.6 Thus, they cannot afford to alienate their colleagues. As an epidemiologist, there is temptation to function primarily as a coffeesipping data analyst, confined mostly to an office, lecturing occasionally to the nursing staff. The message of my editorial was that the physician epidemiologist must teach infection control measures to clinician colleagues despite the inherent risks.

To take that first step and ask physician colleagues to participate in the "fifteen second handwashing drill" requires a measure of courage. Physician epidemiologists deserve the encouragement and support of their nurse counterparts. I make no apologies for the positions taken in my editorial, and-to paraphrase William Osler-hereby write my own epitaph: "I taught handwashing on the wards."

\section{REFERENCES}

I. Bryan CS, Hornung CA, Reynolds KL, et al: Endemic bacteremia in Columbia, South Carolina A m J Epidemiol 1986; 123:113-127.

2. Garner JS, Favero MS: CDC guidelines for handwashing and hospital environmental control, 1985. Infect Control 1986; 7:231-243.

3. Bryan CS, Deever E: Implementing control mea sures. Am J Infect Control 1981; 9:101-106.

4. Bryan CS: The nursing shortage: What can physicians do? (editorial). I SC Med Assoc 1981: 77:455.

5. Ervin FR: The bell colls for the infectious diseases clinician (editorial). J Infect Dis 1986; 153:183-185.

6. Petersdorf RG: Training, cost containment, and practice: Effect on infectious diseases. Rev Infect Dis 1986: 8:478-487.

Charles S. Bryan, MD

Professor of Medicine

Director, Division of Infectious Diseases Richland Memorial Hospital Columbia, SC 


\section{Merck Sharp \& Dohme Research Laboratories... Unlocking the potential of DNA}

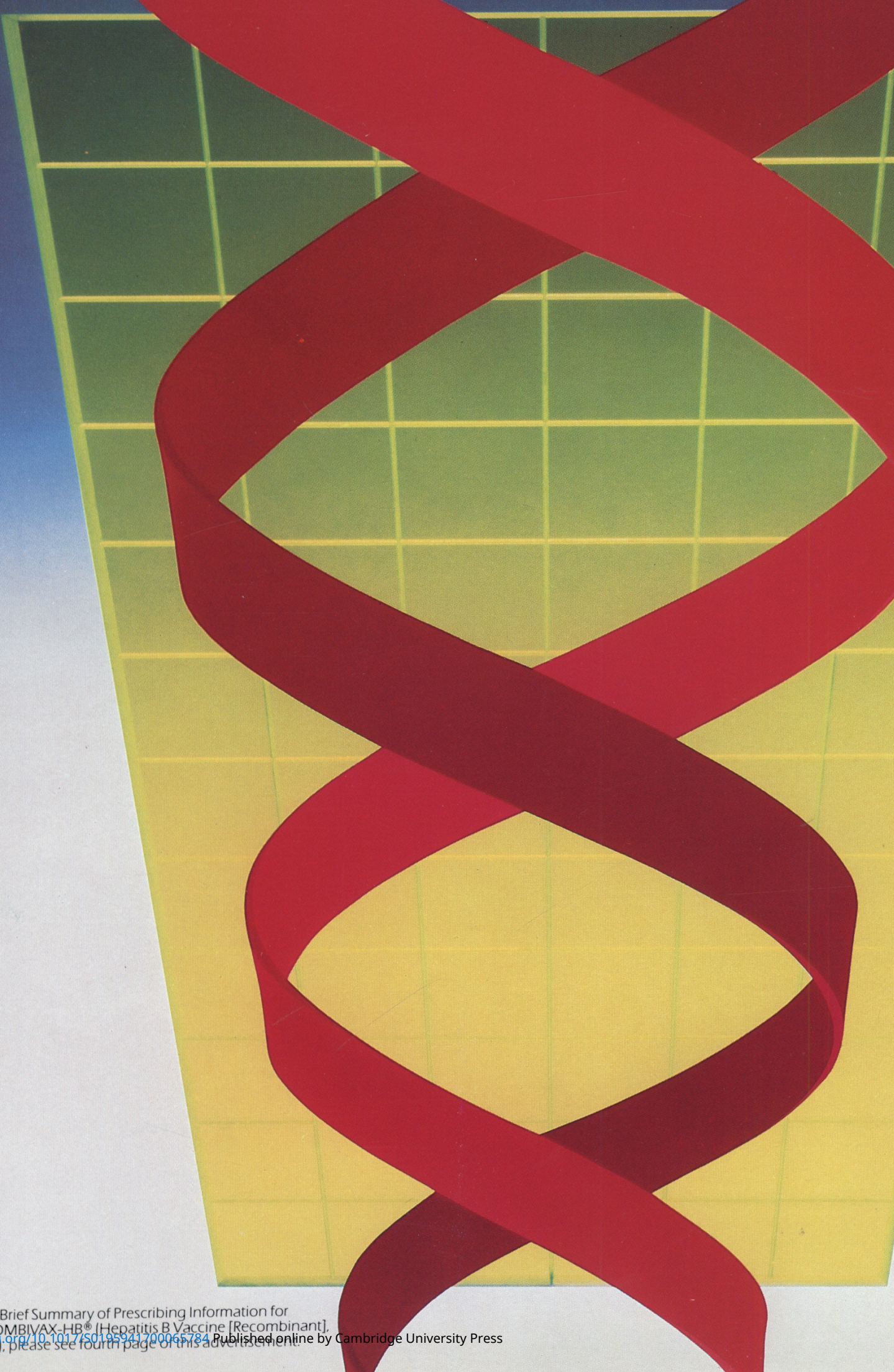




\section{Introducing New}

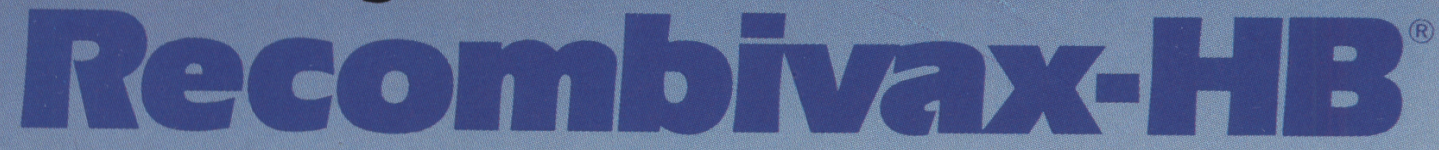

(Hepatitis B Vaccine [Recombinant] |MSD) The first genetically designed vaccine for humans

\section{Another choice}

\section{To help prevent hepatitis B in those at increased risk}

- Derived from yeast by means of advanced biotechnology (not derived from plasma)

- Produces a protective antibody comparable to that of HEPTAVAX-B ${ }^{\circ}$ (Hepatitis B Vaccine, MSD)

- Provides an alternative to vaccination with HEPTAVAX-B

HEPTAVAX-B and RECOMBIVAX-HB are contraindicated in the presence of hypersensitivity to any component of the vaccine.

Patients who develop symptoms suggestive of hypersensitivity after an injection should not receive further injections of HEPTAVAX-B or RECOMBIVAX-HB.

\section{)}

( 


\section{Another chance}

\section{To help protect yourself... your family... and your career}

- Against contracting and transmitting hepatitis B, a potentially serious disease

- Against developing an irreversible chronic-carrier state

\section{To help protect your institution}

- Against the costs of accidental exposure

- Against the devastation of a hepatitis B outbreak

Because of the long incubation period for hepatitis $B$, it is possible for unrecognized infection to be present at the time HEPTAVAX-B ${ }^{\circledast}$ (Hepatitis B Vaccine, MSD) or RECOMBIVAX-HB ${ }^{\circledR}$ (Hepatitis B Vaccine [Recombinant], MSD) is given. HEPTAVAX-B or RECOMBIVAX-HB may not prevent hepatitis B in such patients. 


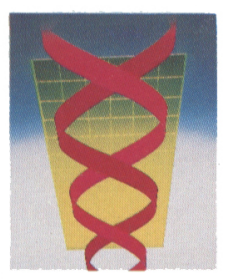

RECOMBIVAX-HB

(Hepatitis B Vaccine [Recombinant]. MSD)

genic in infants and children of all ages Newborns also respond well: maternally transferred antibodies do not interfere with the active immune response to the vaccine See DOSAGE AND ADMINISTRATION for recommended pediatric dosage and for recommended dosage for infants born to HBSAg positive mothers.

\section{ADVERSE REACTIONS}

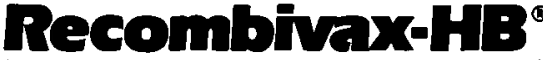

[Hepatitis B Vaccine [Recombinant]|MSD|

INDICATIONS AND USAGE

RECOMBIVAX-HB is indicated for immunization against infection caused by all known subtypes of hepatitis B virus.

RECOMBNAX-HB will not prevent hepatitis caused by other agents, such as hepatitis $A$ virus, non- $A$, non- $B$ hepatitis viruses, or other viruses known to infect the liver.

Vaccination is recommended in persons of all ages who are or will be at increased risk of infection with hepatitis B virus. In areas with high prevalence of infection. most of the population are at risk of acquiring hepatitis B infection at a young age. Therefore, vaccination should be targeted to prevent such transmission. In areas of low prevalence, vaccination should be limited to those who are in groups identified as being at increased risk of infection.

\section{CONTRAINDICATIONS}

Hypersensitivity to yeast or any component of the vaccine.

\section{WARNINGS}

Patients who develop symptoms suggestive of hypersensitivity after an injection should not receive further injections of RECOMBIVAX-HB (see CONTRAINDICATIONS).

Because of the long incubation period for hepatitis B, it is possible for unrecognized infection to be present at the time RECOMBIVAX-HB is given. RECOMBIVAXHB may not prevent hepatitis $B$ in such patients.

\section{PRECAUTIONS}

\section{General}

As with any percutaneous vaccine, epinephrine should be available for immediate use should an anaphylactoid reaction occur.

Any serious active infection is reason for delaying use of RECOMBIVAX-HB except when in the opinion of the physician, withholding the vaccine entails a greater risk.

Caution and appropriate care should be exercised in administering RECOMBIVAX$\mathrm{HB}$ to individuals with severely compromised cardiopulmonary status or to others in whom a febrile or systemic reaction could pose a significant risk.

\section{Pregnancy}

Pregnancy Category C. Animal reproduction srudies have not been conducted with RECOMBIVAX-HB. It is also not known whether RECOMBIVAX-HB can cause fetal harm when administered to a pregnant woman or can affect reproduction capacity. RECOMBIVAX-HB should be given to a pregnant woman only if clearly needed.

\section{Nursing Mothers}

It is not known whether RECOMBIVAX$H B$ is excreted in human milk. Because many drugs are excreted in human milk. caution should be exercised when

RECOMBIVAX-HB is administered to a nursing woman.

\section{Pediatric Use}

RECOMBIVAX-HB has been shown to be usually well tolerated and highly immuno-
RECOMBIVAX-HB is generally well tolerated. No serious adverse reactions attributable to the vaccine have been reported during the course of clinical trials. No serious hypersensitivity reactions have been reported. No adverse experiences were reported during clinical trials which could be related to changes in the titers of antibodies to yeast. As with any vaccine, there is the possibility that broad use of the vaccine could reveal adverse reactions not observed in clinical trials.

In a group of studies, 3258 doses of vaccine were administered to 1252 healthy adults who were monitored for 5 days after each dose. Injection site and systemic complaints were reported following $17 \%$ and

$15 \%$ of the injections, respectively.

The following adverse reactions were reported:

Incidence Equal to or Greater than I\% of Injections

\section{LOCAL REACTION IINJECTION SITE)}

Injection site reactions consisting principally of soreness and including pain, tenderness. pruritus, erythema, ecchymosis, swelling. warmth, and nodule formation.

\section{BODY ASA WHOLE}

The most frequent systemic complaints include fatigue/weakness; headache: fever $1 \geqslant 100^{\circ} \mathrm{Fl}$; malaise

DIGESTIVE SYSTEM

Nausea; diarrhea.

RESPIRATORY SYSTEM

Pharyngitis: upper respiratory infection

Incidence Less than $1 \%$ of Injections

BODY AS A WHOLE

Sweating; achiness; sensation of warmth; lightheadedness; chills; flushing.

DIGESTIVE SYSTEM

Vomiting; abdominal pains/cramps; dyspepsia: diminished appetite.

RESPIRATORY SYSTEM

Rhinitis; influenza: cough.

NERVOUS SYSTEM

Vertigo/dizziness: paresthesia.

INTEGUMENTARY SYSTEM

Pruritus: rash (non-specified); angioedema: urticaria.

\section{MUSCULOSKELETAL SYSTEM}

Arthralgia including monoarticular; myalgia; back pain; neck pain; shoulder pain: neck stiffness.

HEMIC/LYMPHATIC SYSTEM

Lymphadenopathy

PSYCHIATRIC/BEHAVIORAL

Insomnia/disturbed sleep.

SPECIAL SENSES

Earache.

UROGENITAL SYSTEM

Dysuria.

CARDIOVASCULAR SYSTEM

Hypotension.

Potential ADVERSE EFFECTS

In addition, a variety of adverse effects, not observed in clinical trials with RECOMBIVAX-HB have been reported with HEPTAVAX-B ${ }^{\circledR}$ (Hepatitis B Vaccine MSDI (plasma-derived hepatitis B vaccine). Those listed below are to serve as alerting information to physicians:

Hypersensitivity: An apparent hypersensi-

tivity syndrome of delayed onset has been reported days to weeks after vaccination This has included the following findings: arthritis (usually transient), fever, and dermatologic reactions such as urticaria. erythema multiforme, or ecchymoses. Nervous System: Neurological disorders such as optic neuritis myelitis including transverse myelitis; acute radiculoneuropathy including Guillain-Barré syndrome: peripheral neuropathy including Bell's palsy and herpes zoster.

Hematologic: Thrombocytopenia. Special Senses: Tinnitus; visual disturbances.

\section{DOSAGE AND ADMINISTRATION}

Do not inject intravenously or intradermally.

RECOMBIVAX-HB is for intramuscular injection. The deltoid muscle is the preferred site for intramuscular injection in adults. Data suggest that injections given in the buttocks frequently are given into fatty tissue instead of into muscle. Such injections have resulted in a lower seroconversion rate than was expected. The anterolateral thigh is the recommended site for intramuscular injection in infants and young children.

RECOMBIVAX-HB may be administered subcutaneously to persons at risk of hemorrhage following intramuiscular injections. However, when other aluminum-adsorbed vaccines have been administered subcutaneously, an increased incidence of local reactions including subcutaneous nodules has been observed. Therefore, subcutaneous administration should be used only in persons (e.g. hemophiliacs) at risk of hemorrhage following intramuscular injections.

The immunization regimen consists of 3 doses of vaccine. The volume of vaccine to be given on each occasion is as follows

\begin{tabular}{|l|l|lll|}
\hline Group & Formulation & Initial & 1 month & 6 months \\
\hline $\begin{array}{l}\text { Younger Children } \\
\text { (Birth 10 10 } \\
\text { years of age) }\end{array}$ & $\begin{array}{l}\text { Pediatric } \\
5 \mathrm{mcg} / 0.5 \mathrm{~mL}\end{array}$ & $0.5 \mathrm{~mL}$ & $0.5 \mathrm{~mL}$ & $0.5 \mathrm{~mL}$ \\
\hline $\begin{array}{l}\text { Adults and Older } \\
\text { Children }\end{array}$ & $\begin{array}{l}\text { Adult } \\
10 \mathrm{mcg} / 1.0 \mathrm{~mL}\end{array}$ & $1.0 \mathrm{~mL}$ & $1.0 \mathrm{~mL}$ & $1.0 \mathrm{~mL}$ \\
\hline
\end{tabular}

Since there have been no clinical studies in which a vaccine series was initiated with HEPTAVAX-B ${ }^{\circledR}$ (Hepatitis B Vaccine.MSD) and completed with RECOMBIVAX-HB, or vice versa, it is recommended that the 3-dose series be completed with the same vaccine that was used for the initial dose.

Whenever revaccination or administration of a booster dose is appropriate

RECOMBIVAX-HB may be used.

For dosage for infants born of $\mathrm{HBSAg}$ positive mothers and for dosage for known or presumed exposure to HBsAg, see the Prescribing information.

The vaccine should be used as supplied; no dilution or reconstitution is necessary. The full recommended dose of the vaccine should be used.

Storage

Store vials at $2-8^{\circ} \mathrm{C}\left(35.6-46.4^{\circ} \mathrm{F}\right)$. Storage above or below the recommended temperature may reduce potency.

Do not freeze since freezing destroys potency.

For more detalled information, consult your MSD Representative or see Pre. scribing Information. Merck Sharp \& Dohme, Division of Merck \& CO., INC West Point, PA 19486. 


\section{Make a choice... Don't take a chance}
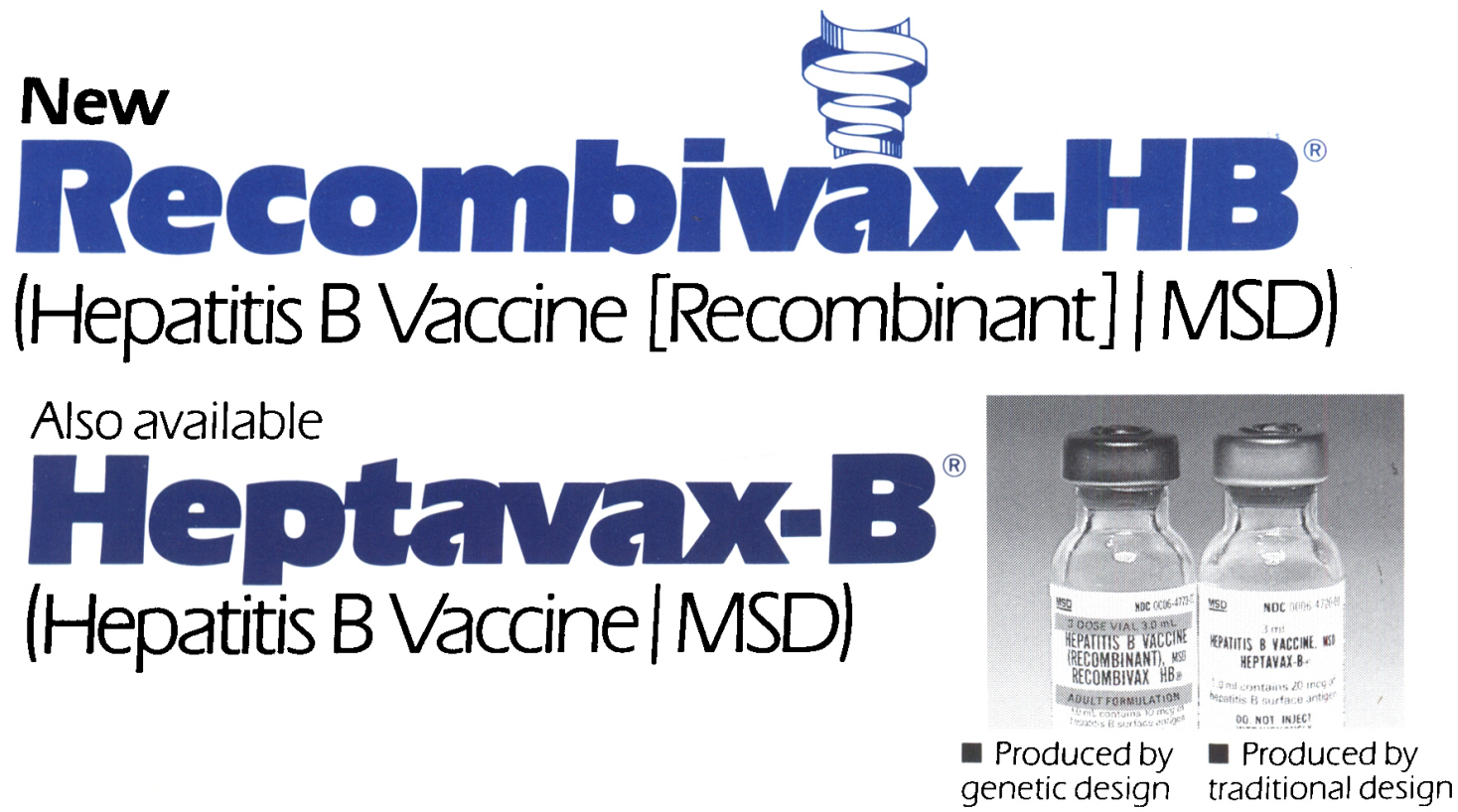

\section{BOTH}

are highly effective against hepatitis $B$ and help prevent its many potential complications and sequelae.

\section{BOTH}

provide highly effective protection for the health-care professional, thereby protecting against possible transmission to family members.

\section{BOTH}

help protect against becoming a chronic carrier and the potential devastating effect on a health-care career.

\section{BOTH}

help protect hospitals against the costly occurrence of a hepatitis B outbreak. 
Heptenax- $\mathbf{B}^{\circ}$

(Hepatitis B Vaccine/MSD)

INDICATIONS AND USAGE

HEPTAVAX-B is indicated for immunization against infection caused by all known subtypes of hepatitis B virus.

HEPTAVAX-B will not prevent hepatitis caused by other agents, such as hepatitis $A$ virus. non- $A$, non- $B$ hepatitis viruses. or other viruses known to infect the liver.

Vaccination is recommended in persons of all ages, especially those who are or will be at increased risk of infection with hepatitis $B$ virus.

\section{CONTRAINDICATIONS}

Hypersensitivity to any component of the vaccine.

\section{WARNINGS}

Persons with immunodeficiency or those receiving immunosuppressive therapy require larger vaccine doses and respond less well than do healthy individuals.

Because of the long incubation period for hepatitis $B$, it is possible for unrecognized infection to be present at the time HEPTAVAX-B is given. HEPTAVAX-B may not prevent hepatitis $B$ in such patients. Patients who develop symptoms suggestive of hypersensitivity after an injection should not receive further injections of HEPTAVAX-B.

\section{PRECAUTIONS}

General

As with any parenteral vaccine, epinephrine should be available for immediate use should an anaphylactoid reaction occur. Any serious active infection is reason for delaying use of this vaccine except when, in the opinion of the physician, withholding the vaccine entails a greater risk. Caution and appropriate care should be exercised in administering this vaccine to individuals with severely compromised cardiopuimonary status or to others in whom a febrile or systemic reaction could pose a significant risk.

\section{Pregnancy}

Pregnancy CategoryC. It is not known whether the vaccine can cause fetal harm when administered to pregnant women or can affect reproductive capacity. The vaccine should be given to a pregnant woman only if clearly needed.

\section{Nursing Mothers}

Studies in 12 lactating women have failed to reveal evidence of this vaccine being secreted in breast milk.

\section{Pediatric Use}

This vaccine has been shown to be well tolerated and highly immunogenic in infants and children of all ages. Newborns also respond well; maternally transferred antibodies do not interfere with the active immune response to the vaccine. See DOSAGE AND ADMINISTRATION section in Prescribing information for recommended pediatric dosage and for recommended dosage for infants born to HBSAg positive mothers.

\section{ADVERSE REACTIONS}

HEPTAVAX-B is generally well tolerated. No serious adverse reactions attributable to vaccination were reported during the course of clinical trials involving administration of HEPTAVAX-B to over 19,000 individ- uals. As with any vaccine, there is the possibility that broad use of the vaccine could reveal rare adverse reactions not observed in clinical triais. In three doubleblind placebo-controlled studies among 3,350 persons, the overall rates of adverse reactions reported by vaccine recipients $(24.3 \%, 21.5 \%$, and $22.8 \%)$ did not differ significantly from those of placebo recipients $(21.4 \%, 18.7 \%$, and $21.9 \%)$. Approximately half of all reported reactions were injection-site soreness, which occurred somewhat more frequently among vaccine recipients

In another group of studies, 3,516 doses of vaccine were administered to 1,255 healthy adults. Vaccinees were monitored for 5 days after each dose, and the following adverse reactions were reported

BODY AS AWHOLE $\%$ of doses

Injection site reactions. consisting principally of soreness, and including erythema, swelling, warmth and induration

Fatigue/asthenia

Malaise

Fever $\left(\geqslant 100^{\circ} \mathrm{F}\right.$

Chills

Sensation of warmth

Irritability

Diaphoresis

DIGESTIVE SYSTEM

Gastrointestinalillness including anorexia, nausea. vomiting abdominal pain and diarrhea

Abdominal cramps

HEMATOLOGIC AND LYMPHATIC SYSTEM

MUSCULOSKELETAL SYSTEM

Myalgia

Arthralgia

NERVOUS SYSTEM

Headache

Dizziness

Disturbed sleep

Paresthesia

RESPIRATORY SYSTEM

Upper respiratory illness

INTEGUMENTARY SYSTEM

Rash (non-specific

In a double-blind placebo-control

clinical trial of 1,330 health-care workers, the frequency of elevations in SGPT (ALT) in vaccine recipients was not significantly different from that in placebo recipients. in marketed use of the vaccine, non-specific abnormalities in SGPT (ALT) and other liver function tests have been reported, but no causal relationship has been established.

The following additional adverse reactions have been reported with use of the marketed vaccine.

Hypersensitivity Reaction

Symptoms of immediate hypersensitivity including urticaria, angioedema, and pruritus have been reported rarely within the first few hours after vaccination. An apparent hypersensitivity syndrome of delayed onset has been reported rarely, days to weeks after vaccination. This has included the following findings: arthritis (usually transient), fever. and dermatologic reactions such as urticaria. erythema multiforme, or ecchymoses. Nervous System

Neurological disorders such as: optic neuritis: myelitis, including transverse myelitis; acute radiculoneuropathy, including Guillain-Barré syndrome: peripheral neuropathy, including Bell's palsy and herpes zoster.

Hematologic

Thrombocytopenia.
HEPTAVAX-B ${ }^{\circ}$

(Hepatitis B Vaccine. MSD)

Special Senses

Tinnitus visual disturbances. Integumentary System

Flushing

DOSAGE AND ADMINISTRATION

Do not inject intravenously or intradermally.

HEPTAVAX-B is for intramuscular injection. The deltoid muscle is the preferred site for intramuscular injection in adults. Data suggest that injections given in the buttocks frequently are given into fatty tissue instead of into muscle. Such injections may result in a lower seroconversion rate than is expected. The anterolateral thigh is the recommended site for intramuscular injection in infants and children.

HEPTAVAX-B may be administered subcutaneously to persons at risk of hemorrhage following intramuscular injections. The immune responses and clinical reactions following intramuscular and subcutaneous administration of HEPTAVAX-B have been shown to be comparable. However, when other aluminum-adsorbed vaccines have been administered subcutaneously, an increased incidence of local reactions including subcutaneous nodules has been observed. Therefore, subcutaneous administration should be used only in persons (e.g. hemophiliacs) at risk of hemorrhage following intramuscular injections.

Shake well before withdrawal and use. Thorough agitation at the time of administration is necessary to maintain suspension of the vaccine.

Parenteral drug products should be inspected visually for particulate matter and discoloration prior to administration.

The immunization regimen consists of 3 doses of vaccine. The volume of vaccine to be given on each occasion is as follows:

\begin{tabular}{|c|c|c|c|c|}
\hline Group & Formulation & Initial & 1 month & 6 months \\
\hline $\begin{array}{l}\text { Younges Children } \\
\text { [Burth :o } 10 \\
\text { years of age) }\end{array}$ & $\begin{array}{l}\text { Pediatric } \\
10 \mathrm{mcg} / 0.5 \mathrm{ml}\end{array}$ & $05 \mathrm{~mL}$ & $0.5 \mathrm{~mL}$ & $0.5 \mathrm{~mL}$ \\
\hline $\begin{array}{l}\text { Adulls and Older } \\
\text { Children }\end{array}$ & $\begin{array}{l}\text { Adult } \\
20 \mathrm{mcg} / 1.0 \mathrm{~mL}\end{array}$ & $1.0 \mathrm{~mL}$ & $10 \mathrm{mt}$. & $1.0 \mathrm{~mL}$ \\
\hline $\begin{array}{l}\text { Oralysis Palients } \\
\text { and Immuno- } \\
\text { compromised } \\
\text { Patients }\end{array}$ & $\begin{array}{l}\text { Adult } \\
20 \mathrm{mcg} / 1.0 \mathrm{~mL}\end{array}$ & $2.0 \mathrm{~mL} \mathrm{~L}^{*}$ & $2.0 \mathrm{~mL}{ }^{*}$ & $2.0 \mathrm{~mL}^{*}$ \\
\hline
\end{tabular}

For dosage for infants born of $\mathrm{HBsAg}$ positive mothers and for dosage for known or presumed exposure to HBsAg. see the Prescribing Information.

The vaccine should be used as supplied: no dilution or reconstitution is necessary. The full recommended dose of the vaccine should be used.

Storage

Store vials at $2-8^{\circ} \mathrm{C}\left(35.6-46.4^{\circ} \mathrm{F}\right)$. Storage above or below the recommended temperature may reduce potency.

Do not freeze since freezing destroys potency.

For more detailed information, consult your MSD Representative or see Prescribing Information. Merck Sharp \& Dohme, Division of Merck \& CO.. INC West Point, PA 19486. 